\title{
Talbot carpets by rogue waves of extended nonlinear Schrödinger equations
}

\author{
Stanko N. Nikolić $($ Omar A. Ashour • Najdan B. Aleksić • \\ Yiqi Zhang • Milivoj R. Belić • Siu A. Chin
}

Received: 6 March 2019 / Accepted: 30 May 2019 / Published online: 15 June 2019

(C) The Author(s) 2019

\begin{abstract}
We present analytical and numerical doubleperiodic solutions of the one-dimensional nonlinear Schrödinger equation and its extended versions in the form of Talbot carpets. The breathers and rogue waves of different orders are obtained using numerical simulations, starting from the initial conditions calculated by the Darboux transformation. To suppress undesirable aspects of modulation instability leading to homoclinic chaos, Fourier mode pruning procedures are invented to preserve and maintain the twofold periodicity of carpets. The novelty of this paper is analytical Talbot carpets for Hirota-quintic equation and ability to obtain them dynamically by controlling the growth of the Fourier modes. In addition, the new period-matching procedure is also described for periodic rogue waves that can be utilized to produce Talbot carpets without
\end{abstract}

S. N. Nikolić $(\varangle) \cdot$ N. B. Aleksić · M. R. Belić Science Program, Texas A\&M University at Qatar, P.O. Box 23874, Doha, Qatar

e-mail: stankon@ipb.ac.rs

S. N. Nikolić · N. B. Aleksić

Institute of Physics Belgrade, University of Belgrade,

Pregrevica 118, Belgrade 11080, Serbia

O. A. Ashour

Department of Physics, University of California, Berkeley, Berkeley, CA 94720, USA

\section{Y. Q. Zhang}

Department of Applied Physics, School of Science, Xi' an Jiaotong University, Xi' an 710049, China

\section{S. A. Chin}

Department of Physics and Astronomy, Texas A\&M University, College Station, TX 77843, USA mode pruning. Tablot carpets may find future utility in optoplasmonic nanolithography.

Keywords Quintic equation · Talbot carpet · Rogue waves

\section{Introduction}

Nonlinear Schrödinger equations (NLSEs) of different orders continue to elicit acute attention of numerous research groups around the world, for their utility in various branches of mathematics and physics [1-8]. Here, our attention is focused on the one-dimensional NLSEs arising in the extension of the basic cubic NLSE up to the fifth-order in nonlinearity and dispersions, of utility in nonlinear fiber optics. In particular, we are interested in the unstable solutions of these models when the modulation instability sets in.

Modulation instability (MI) is the basic nonlinear optical process in which a weak periodic perturbation of the fundamental pump wave produces an exponential growth of a finite number of spectral sidebands locked to and growing at the expense of the pump $[4,5,9]$. Although commonly known as the BenjaminFeir instability of Stokes waves, that appeared in the 1960s, the MI of the cubic NLSE debuted already in 1947, in the Bogoliubov's work on the uniform Bose gas [10]. It is widely believed that MI is the root cause of the appearance of rogue waves (RWs) in nonlinear optics. The problem is, how to systematically incorpo- 
rate the process of MI into the dynamics of generation and observation of RWs in the NLSEs of different types.

A convenient handle in this process is provided by the existence of a family of exact solutions to the basic cubic NLSE in the form of Akhmediev breathers (ABs), Kuznetsov-Ma (KM) solitons, and the Peregrine soliton, which may be regarded as the elementary solutions on a finite background from which higher-order RW solutions can be formed. In this sense, ABs seem to be especially relevant $[11,12]$, which can be generalized to the doubly periodic solutions (as well as to the extended NLSEs). They allow for an easy systematic buildup of higher-order breathers that can be regarded as prototype RWs $[13,14]$.

The complication is that these basic and higherorder solutions represent homoclinic orbits of unstable Stokes waves in the dynamics of cubic NLSE [15-18]. The generic long-time dynamics of modulated Stokes waves, for example, ABs with two or more unstable modes, is chaotic. Once the system, for a range of relevant parameters and initial and boundary conditions, enters homoclinic chaos, the predictive power of the model diminishes. The question has even been raised whether the chaos seen belongs to the model itself or is induced by the numerical procedure applied $[15,16]$.

For these reasons, a school of thought has emerged which holds that it is not important to follow exact dynamics of individual members of the family of exact solutions, but to look at the statistics of RWs in the chaotic regime $[4,6,13,19,20]$. Optical RWs are rare extreme events in the fluctuation of optical fields; therefore, their statistical features, such as long-tailed probability distributions, should be considered as their defining features. Thus, one should proceed with the numerical solution of different NLSEs with appropriate initial conditions seeded with noise of various types, and after many runs compare the associated statistics of the resulting field distributions with the available experimental data.

In this paper, we adopt a different approach. It is our belief that for specific applications, one still must perform carefully designed numerical simulations of individual well-defined RW solutions, even when it leads to following their dynamics deep in the chaotic region. The general idea is to discern order from chaos. The specific goal is to investigate the possibility of producing Talbot carpets out of ABs of different orders, with an eye on possible applications in nanolithography. Such an investigation requires launching an exact breather and following its repeated self-imaging recurrences for as long as possible.

Thus, we examine how AB and RW solutions may be obtained for the NLSE and its extensions, the Hirota and quintic equation, and used to accomplish the goal stated. To this end, of immense importance are the analytical solutions to the NLSE that are periodic both along the spatial and temporal axes, and can be viewed as Talbot self-images, introduced in [12]. This study was extended in [21,22], where the nonlinear Talbot carpets of rogue waves were reported for the first time. These solutions are associated with the Talbot effect, first described in the nineteenth century [23], about the same time the solitary waves were discovered. An interesting feature of the nonlinear Talbot effect is that it only displays the primary and secondary images.

The Talbot effect is a near-field diffraction effect, observed when light beams diffract at some periodic structure (such as gratings) and produce recurrent selfimages at equidistant planes. In-between the planes, fractional and even fractal images are observed, leading to intricate light patterns that are called the Talbot carpets. Later, the self-imaging phenomena have been reported in many areas of physics, such as atomic [24,25] and quantum [26] optics, waveguide arrays [27], Bose-Einstein condensates [28,29], photonic lattices [30], and X-ray imaging [31]. Talbot self-images can even be regarded as an example of Fermi-PastaUlam recurrence [9]. Nonlinear Talbot effect from nonlinear photonic crystals was experimentally demonstrated in [32]. An overview of the recent advances of Talbot effect in modern science is presented in [33].

The major advances in this paper can be stated as follows. We present the dynamical generation of breathers and rogue waves in Talbot carpet-like arrangements, for the NLSE and its extensions. We calculate the firstand higher-order breathers using Darboux transformation (DT) and extract initial conditions in a wide box that is a multiple of the main breather's period [34]. We invent two pruning procedures for Fourier modes, to suppress modulation instability that ruins the doubleperiodic pattern of high-intensity peaks. We generalize the NLSE solution formula from [12] and obtain exact solutions for the Hirota-quintic equation. We display a new class of solutions and analyze different mode pruning algorithms for their dynamical stabilization. We introduce a novel way for generating Talbot carpets for the quintic equation on nonuniform backgrounds, 
based on specific ratios of the breather and elliptic background periods on which the breathers ride.

The paper is organized in the following fashion. In Sect. 2, we introduce the model of generalized NLSEs and analyze a numerical algorithm for generating nonlinear Talbot carpets from their solutions that may or may not require mode pruning. In Sect. 3, we provide exact solutions of the Hirota-quintic equation and exhibit dynamical procedure for producing Talbot carpets for this specific extended NLSE. In Sect. 4, we build double-periodic RWs on a dnoidal background for the quintic equation and arrange them also in the form of Talbot carpets. In Sect. 5, we summarize our results.

\section{Dynamical double-periodic solutions of the extended NLSE}

In this paper, we study double-periodic solutions of the extended nonlinear Schrödinger equation, called the quintic NLS equation (QNLSE)

$$
\begin{aligned}
& i \psi_{x}+S[\psi(x, t)]-i \alpha H[\psi(x, t)] \\
& \quad+\gamma P[\psi(x, t)]-i \delta Q[\psi(x, t)]=0 .
\end{aligned}
$$

Here, the transverse variable is denoted by $t$ and the longitudinal variable by $x$, while $\alpha, \gamma$ and $\delta$ are arbitrary real numbers used to introduce higher-order terms. The wave function $\psi \equiv \psi(x, t)$ represents the slowly varying envelope that could be optical, plasmonic or other in nature. A distinct value of Eq. (1) is that it represents a general extension of the NLS equation to the quintic order that includes in a consistent manner various dispersive and nonlinear contributions of relevance to the propagation of pulses in fibers.

Operators $S, H, P$, and $Q$ comprise the nonlinearity terms and higher-order spatial dispersions. They arise in an infinite hierarchy of NLSEs $[7,8,35]$. Their expressions are as follows:

$$
\begin{aligned}
S[\psi(x, t)]= & \frac{1}{2} \psi_{t t}+|\psi|^{2} \psi \\
H[\psi(x, t)]= & \psi_{t t t}+6|\psi|^{2} \psi_{t}, \\
P[\psi(x, t)]= & \psi_{t t t t}+8|\psi|^{2} \psi_{t t}+6|\psi|^{4} \psi+4\left|\psi_{t}\right|^{2} \psi \\
& +6 \psi_{t}^{2} \psi^{*}+2 \psi^{2} \psi_{t t}^{*},
\end{aligned}
$$

$$
\begin{aligned}
Q[\psi(x, t)]= & \psi_{t t t t t}+10|\psi|^{2} \psi_{t t t} \\
& +30|\psi|^{4} \psi_{t}+10 \psi \psi_{t} \psi_{t t}^{*} \\
& +10 \psi \psi_{t}^{*} \psi_{t t}+20 \psi^{*} \psi_{t} \psi_{t t}+10 \psi_{t}^{2} \psi_{t}^{*},
\end{aligned}
$$

where the subscripts $t$ [and $x$ in Eq. (1)] represent the partial derivatives. Additional terms of higher-order dispersion and nonlinearity are required for the description of ultrashort pulse propagation through optical fibers [2,36-39].

The third-order term (Hirota) is used to explain the generation of supercontinuum [5] and pulse-deforming phenomena in fibers [40]. The fourth-order (Lakshmanan-Porsezian-Daniel) operator appears in the analysis of Heisenberg spin chains [35]. The fifthorder dispersion (quintic) is noticeable in laser experiments with ultrashort pulse duration (below $20 \mathrm{fs}$ ) [41].

When $\alpha=\gamma=\delta=0$, Eq. (1) reduces to the wellknown cubic NLSE, which is the fundamental equation of nonlinear optics $[4,9,20,42,43]$. If only $\alpha \neq 0$, we deal with the Hirota equation [44-47]. In case of nonzero $\alpha$ and $\delta$ with $\gamma=0$, we talk about Hirotaquintic equation.

Various solutions of the QNLSE, such as solitons [48,49], breathers [49,50], and RWs [51], have been discussed in the literature. It is well known that breathers and solitons of arbitrary order can be obtained analytically using the DT technique. These solutions are single periodic: breathers along $t$, and solitons along $x$ direction. Single-periodic NLSE solutions, such as ABs, can be utilized to dynamically construct nonlinear Talbot carpets, which are also intimately connected with the double-periodic solutions of NLSE.

The period of an $\mathrm{AB}$ (first order or higher order) is determined by the single parameter $a<0.5$ of the solution [9]:

$L=\frac{\pi}{\sqrt{1-2 a}}$.

The initial condition for dynamical generation is derived from exact $\mathrm{AB}$ solutions at a certain value of the evolution variable $x=x_{0}$, using Darboux transformation [52]. Here, it is essential to adjust the size of the transverse box $\left(t_{1}, t_{2}\right)$ to an integer multiple $M$ of the fundamental breather's period and apply periodic boundary conditions,

$\Delta T=t_{2}-t_{1}=M L$. 
(a)

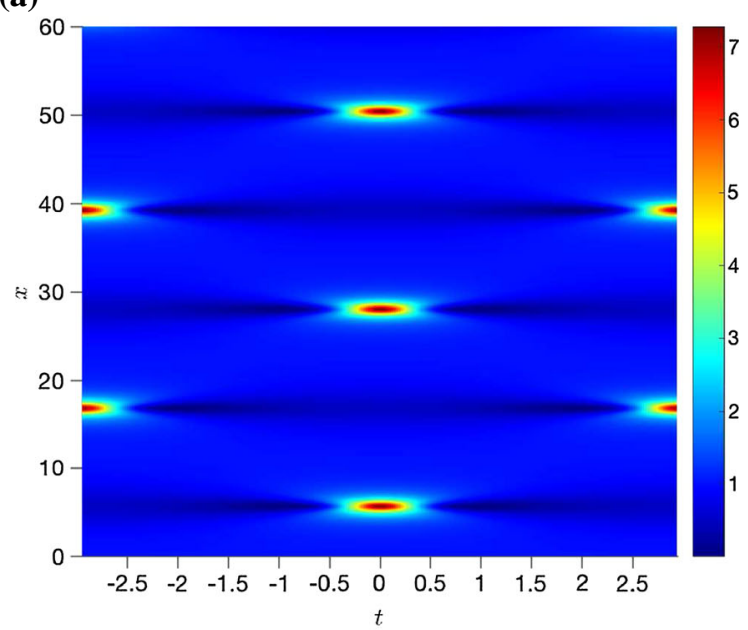

(c)

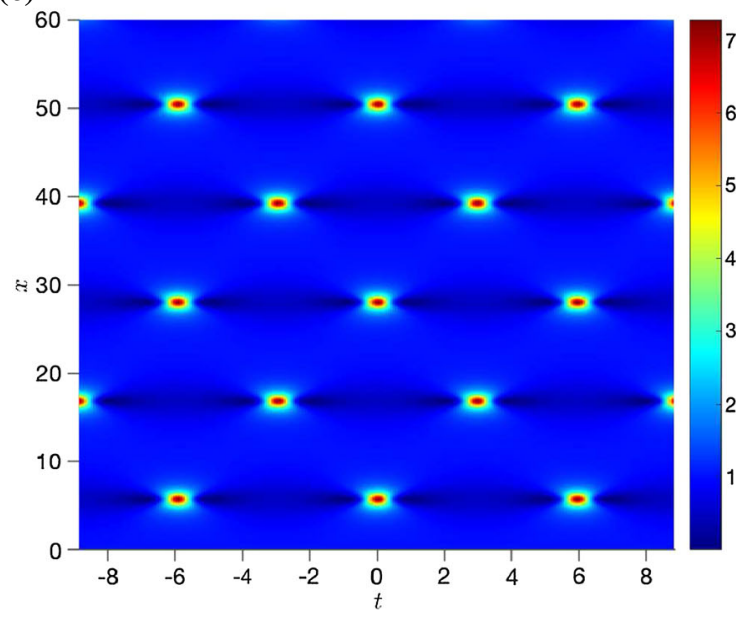

Fig. 1 Double-periodic numerical solutions, made of the firstorder NLSE breathers $(\alpha=\gamma=\delta=0)$, using the pruning procedure in FFT. The breather parameter is $a=0.36$. a One

Numerical solutions of NLSEs in this paper are obtained using the second-order split-step fast Fourier transform (FFT) method. When the box size is exactly equal to the breather's fundamental period $L$, the Fourier harmonics form the basic set of spatial frequencies

$$
S_{1}=\left\{\omega_{j}=j \Omega ; \mid 0 \leq j<N\right\},
$$

where $\Omega=2 \pi / L$ is the mode spacing and $N$ the total number of modes. The mode growing out of this basic set will be the stable fundamental breather mode. However, if the box is larger $(M>1)$, the fundamental (b)

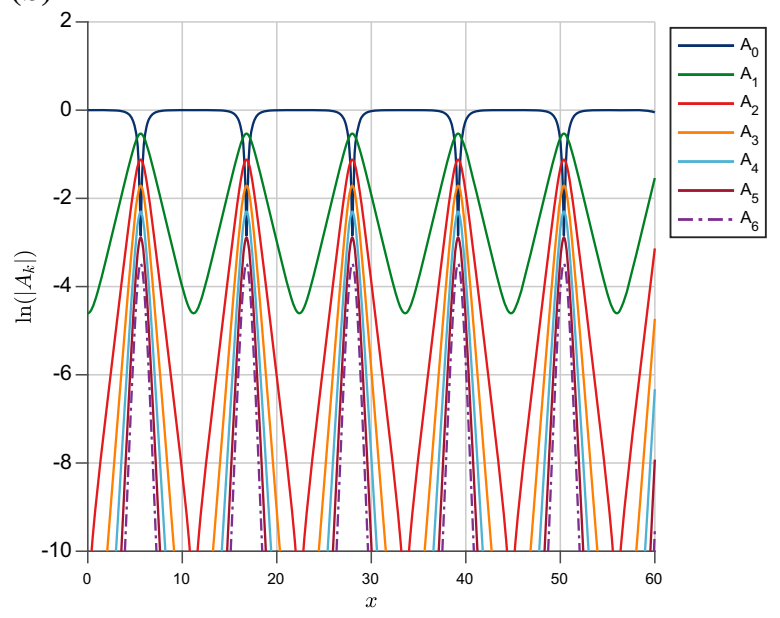

(d)

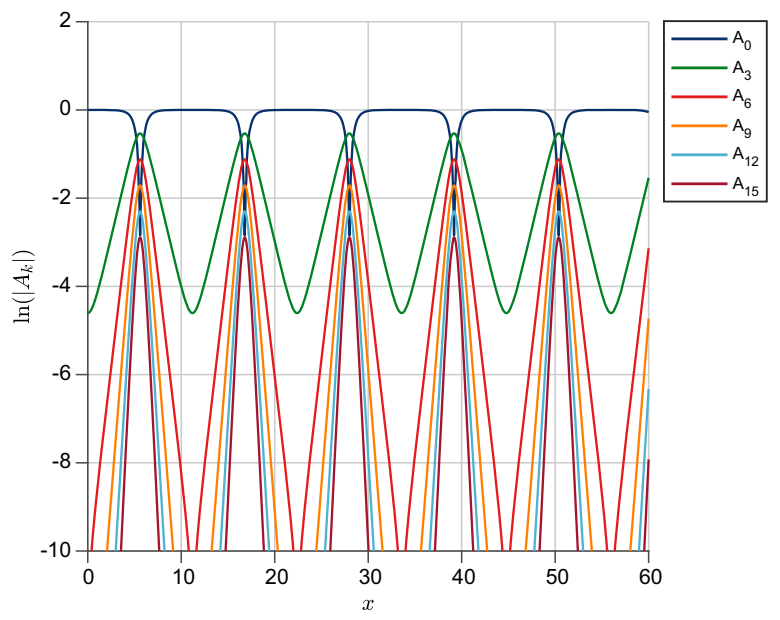

breather in the box, no pruning. $\mathbf{b}$ Its spectrum. $\mathbf{c}$ Three breathers ( 3 periods) in the box, with the pruning. $\mathbf{d}$ The corresponding spectrum

mode and mode spacings are smaller $\Omega_{M}=\Omega / M$, and Fourier modes form a new set $S_{M}$ with a larger number of modes $(N M)$. All modes from $S_{M}$ that are not elements of $S_{1}$ exponentially grow from infinitesimal amplitudes, owing to modulation instability. Now, the modes from $S_{1}$ are also under MI and grow exponentially, but they interfere constructively and only form the fundamental $\mathrm{AB}$ mode.

The key point in generating nonlinear Talbot carpets is to suppress the undesirable unstable Fourier modes. This can be achieved in different ways. In the simplest, after each numerical iteration one simply eliminates the unstable subharmonics, leaving only the ones responsi- 
(a)
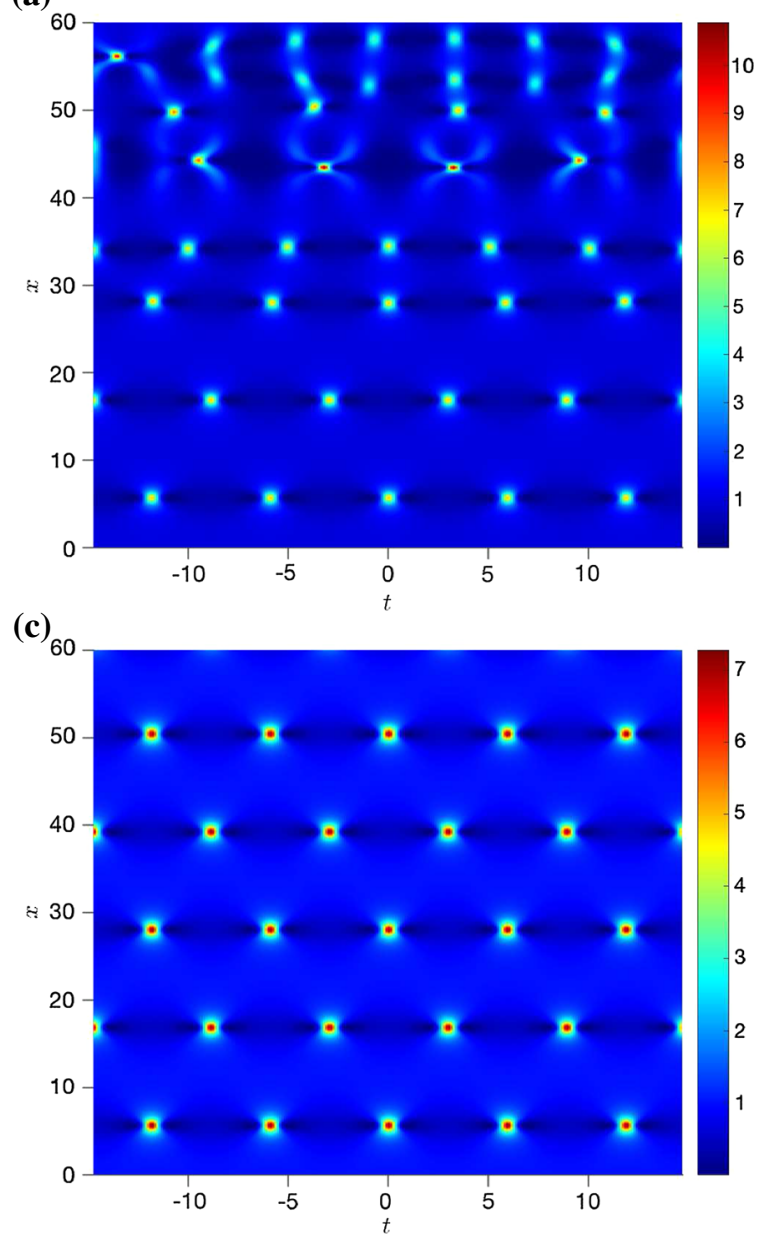

Fig. 2 Dynamical generation of Talbot carpet from the firstorder breathers using a DT initial condition, with $a=0.36$ and $M=5$. a A failed attempt, due to MI. b The corresponding Fourier spectrum, displaying loss of Talbot periodicity due to

ble for the formation of the fundamental $\mathrm{AB}$ (labeled as $0, \pm M, \pm 2 M, \pm 3 M$, etc). It effectively eliminates MI. The procedure is illustrated in Fig. 1. Although quite drastic, it apparently works. The other ways include suppressing the unstable modes selectively and to a degree.

In Fig. 1a, we show numerical evolution of the firstorder Akhmediev breather $(a=0.36)$ when the box size is equal to the breather's period $(M=1)$. One can see that the intensity peak at $t=0$ is repeated along $x$ axis at the Talbot periods, forming a stable mode. This peak is consecutively shifted for half a period along $t$-axis, forming the secondary Talbot image at half the (b)

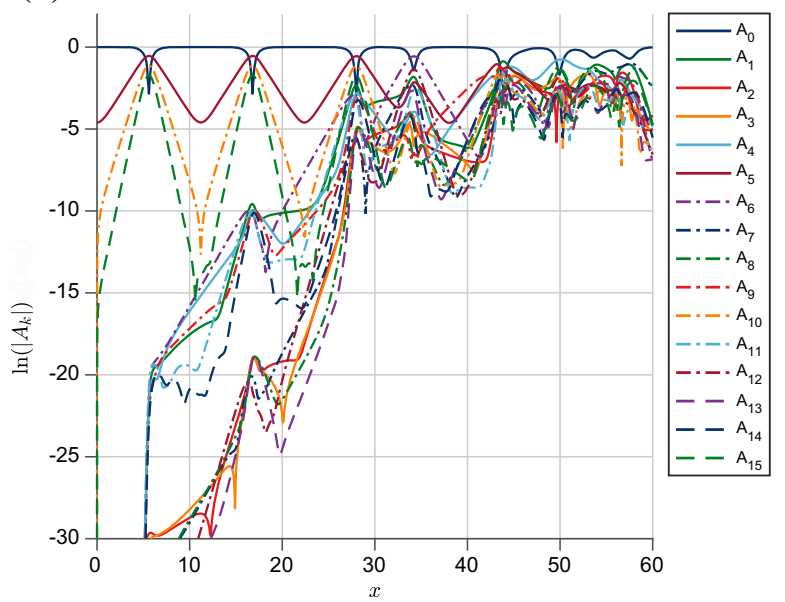

(d)

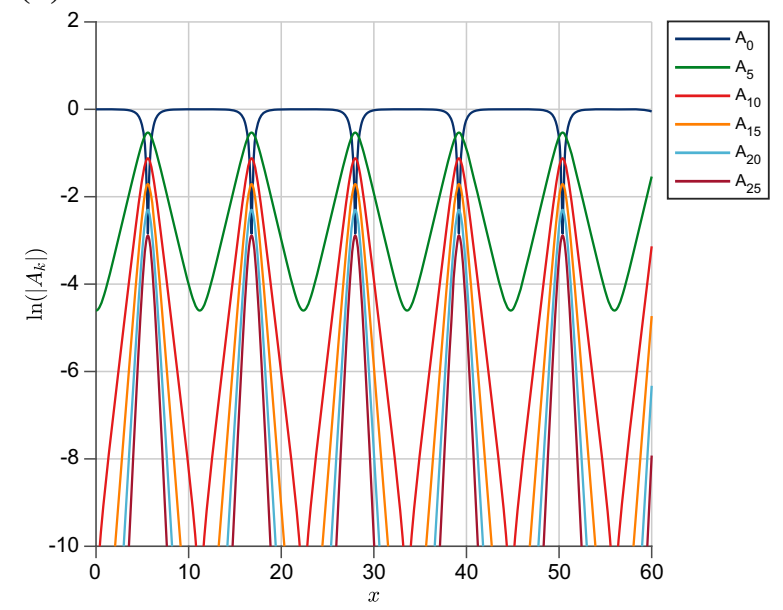

the exponential growth of non-quintuplet modes. c Successful generation of the Talbot carpet using the pruning procedure. $\mathbf{d}$ Fourier spectrum after the pruning is applied

Talbot period. The corresponding Fourier spectrum is shown in Fig. 1b. Next, we calculate the same breather over three periods $(M=3)$. We apply the simple pruning algorithm to Fourier modes, setting all unstable mode amplitudes to zero except the triplet modes, indexed as $0, \pm 3, \pm 6, \pm 9$, and so on. The result is an extended Talbot carpet with alternate shifting of intensity maxima along $x$ - and $t$-axes, as presented in Fig. $1 \mathrm{c}$. The spectrum of the triplet mode amplitudes is shown in Fig. 1d.

In Fig. 2, we display how the simple pruning technique actually works. We again choose the first-order breather with $a=0.36$ and set the numerical box to 
(a)

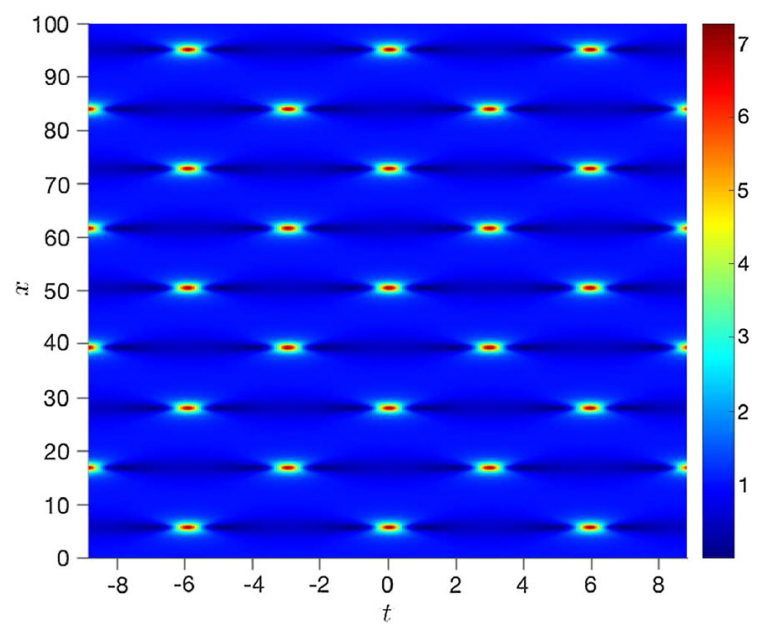

(b)

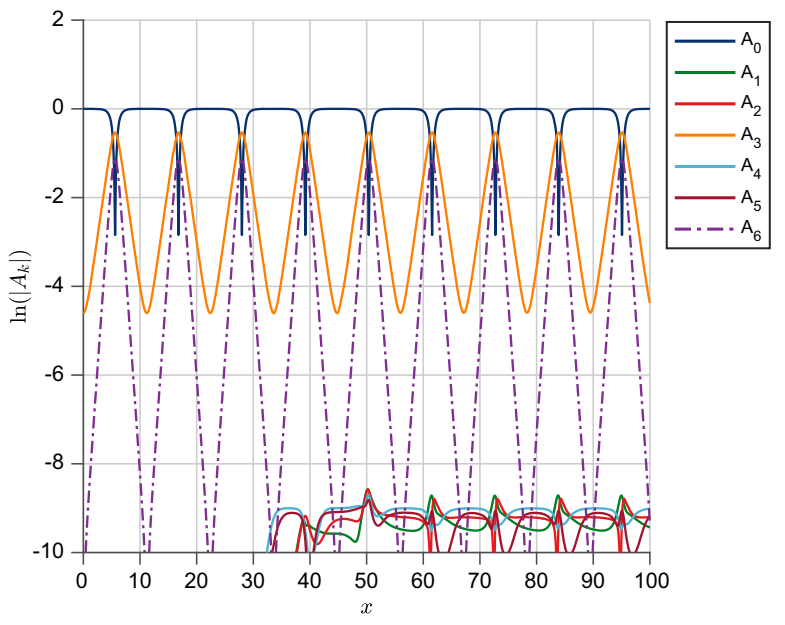

Fig. 3 Same as Fig. 1 but with Gaussian pruning. a The carpet. b Its spectrum. The modes at the bottom are the suppressed unstable modes

be exactly five times the breather's period: $M=5$. In this case, the $\mathrm{AB}$ will be formed by the modes $A_{0}, A_{5}, A_{10}, \ldots, A_{5 m}$. If the pruning algorithm is not applied, the chaotic behavior ruins the carpet after just one full Talbot cycle, as shown in Fig. 2a. This is the MI in action: The unstable modes grow exponentially and prevent the homoclinic orbit (the initial $\mathrm{AB}$ mode) from returning to itself after more than one cycle. This is clearly observed in the buildup of Fourier spectrum of all modes (Fig. 2b), which destroys the spatial Talbot periodicity. Note that after the full cycle, another displaced $\mathrm{AB}$ appears but not at half-cycle, interacting

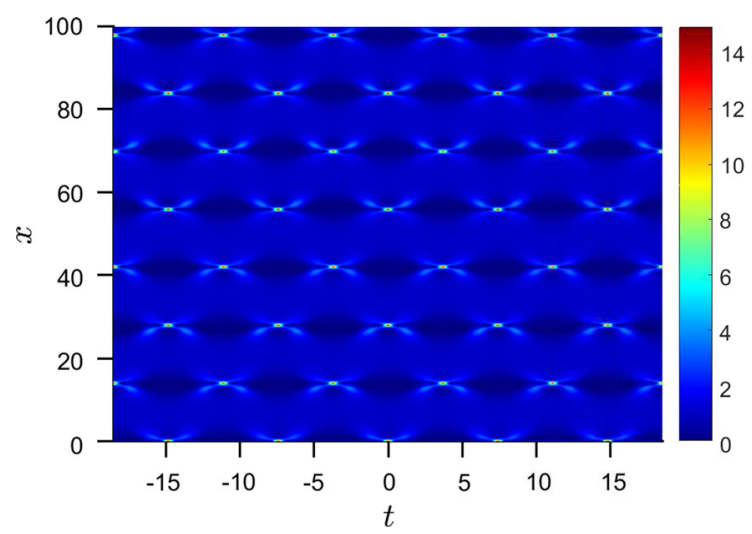

Fig. 4 Double-periodic numerical solution of NLSE, made of the second-order breathers, having $a=0.41$. The box contains 5 breather's periods. The solution is obtained using the pruning procedure with the full mode. As a result of this interaction, or beating of the two modes, two second-order ABs (that can be regarded as the second-order RWs) are formed around $x=45$, which constitutes the normal channel for the production of RWs through MI. However, when the pruning procedure is applied, all non-quintuplet modes are killed after each iteration. Effectively, the procedure prevents the orbit to wander in the homoclinic tangle, forcing it to stick to itself and return back to the starting point. The result is the perfect nonlinear Talbot carpet (Fig. 2c), with the perfect Fourier spectrum (Fig. 2d).

In Fig. 3, we illustrate the Gaussian pruning algorithm, in which the unstable modes are not eliminated completely but suppressed by a Gaussian factor. Thus, the unstable modes are multiplied by a Gaussian factor that depends on their strength: When weak, they are allowed to grow; but the more they grow the more they are suppressed. Effectively, they can grow only up to a certain level, determined by the Gaussian distribution. In Fig. 3b, the unstable modes, visible at the bottom of the figure, cannot grow above the level of approximately $10^{-9}$.

In Fig. 4, we present the nonlinear Talbot carpet consisting of the second-order breathers with consequently higher peak intensity. It can be regarded as a carpet composed of rogue waves. The box size is five times the fundamental breather period, having $a=0.41$. This breather contains two unstable modes. Initial con- 
ditions were derived from DT along a line passing through the breather's maximum. As in the previous figures, the simple pruning algorithm was used, which left only the quintuplet Fourier modes intact.

Obtaining Talbot carpets composed of the thirdorder $\mathrm{ABs}$ required tedious mode elimination procedure, due to inherent instability of such solutions and low probability of the triple collisions of breather's constituents in the $x t$-plane. No effective pruning algorithm could be devised yet, although different mode pruning techniques produced nonuniform carpets of extended stability. We are actively engaged in this investigation, and hope to resolve the issues in a future publication.

\section{Talbot carpets for the Hirota and Hirota-quintic equation}

A family of double-periodic solutions of NLSE that include Jacobi elliptic functions (JEFs) is presented in [12] and in Eq. (6) of [21]. Here, we report the generalization of this expression to the exact solution of the Hirota-quintic equation:

$$
\begin{aligned}
& \psi(x, t) \\
& =\frac{k}{\sqrt{2}} \frac{A(x, t) \cdot \operatorname{dn}\left(\frac{k x}{2}, g=\frac{1}{k}\right)+\frac{i}{k} \operatorname{sn}\left(\frac{k x}{2}, g=\frac{1}{k}\right)}{1-A(x, t) \cdot \operatorname{cn}\left(\frac{k x}{2}, g=\frac{1}{k}\right)} . \\
& \cdot e^{i x / 2},
\end{aligned}
$$

where

$$
\begin{aligned}
& A(x, t) \\
& =\frac{\operatorname{cn}\left(\sqrt{k}\left(t+2 \alpha x+\left(4-\frac{1}{2} k^{2}\right) \delta x\right), g=\sqrt{\frac{k-1}{2 k}}\right)}{\sqrt{1+k}}
\end{aligned}
$$

$g$ is the elliptic modulus, $m=g^{2}$ is the elliptic modulus squared, and $k$ is a positive real constant. The JEF functions $\operatorname{sn}(x, g), \operatorname{cn}(x, g)$, and $\operatorname{dn}(x, g)$ are all periodic, with the periods $4 K, 4 K$, and $2 K$, respectively, where $K(m)=\int_{0}^{\pi / 2} d \theta / \sqrt{1-g^{2} \sin ^{2} \theta}$ is the complete elliptic integral of the first kind. Therefore, solutions described in Eq. (9) are periodic in both $t$ and $x$ directions for any $k>0$. They allow the formation of Talbot carpets for the extended NLSE, up to the fifthorder dispersion.
We use analytical solution from Eq. (9) to dynamically generate Talbot carpets for the Hirota equation $(\alpha \neq 0, \gamma=\delta=0)$. We calculate initial conditions for numerics at some particular $x$ value using Eq. (9). However, the numerical algorithm is different from the basic NLS equation. Since we have $6|\psi|^{2} \psi_{t}$ terms in the $H$ operator, comprising both dispersion and nonlinearity in a single term, it is not convenient to use FFT. Instead, we use a finite difference method to calculate derivatives and the fourth-order explicit Runge-Kutta method for the evolution of the wave function.

In Fig. 5a, we show a failed attempt to dynamically generate Hirota Talbot carpet $(\alpha=0.2712, \gamma=\delta=0$, and $k=1.7286)$. The box is exactly equal to 10 periods along the $t$-axis. At $x \approx 25$, modulation instability starts to ruin the carpet, leading to chaotic behavior of the modes. The intensity peaks are smeared and of lower intensity, since the unstable modes increase their amplitudes during evolution. To overcome the influence of MI, after each few iterations we perform the FFT of $\psi(x, t)$ and then set the amplitudes of all modes to zero, except for the modes with indices $0, \pm 10$, $\pm 20, \pm 30$, etc. In this manner, modulation instability is suppressed and Talbot carpet produced, as shown in Fig. 5b. The numerical solution obtained in this way is equal to the analytical one, all the way to the total calculation time $(x=60)$.

In the next example, we exhibit analytical Talbot carpets of the Hirota-quintic equation, having $\gamma=0$ and $k=1.772$. No pruning is needed here. In Fig. 6a, we show Talbot carpet obtained for $\alpha=-0.2712$ and $\delta=-0.1$. In general, the breathers are tilted. One can infer that the tilt of each breather-like structure to the right is caused by the negative signs of quintic parameters. If signs of $\alpha$ and $\delta$ are changed, identical intensity patterns will be produced, but with the tilt in the opposite direction (Fig. 6b). If $\alpha$ and $\delta$ have different signs, then Hirota and quintic terms will tend to tilt and stretch intensity maxima in different directions. For the given values of $\alpha$ and $\delta$, one can balance the action of $H$ and $Q$ operators, and obtain a solution that satisfies both the NLSE and Hirota-quintic equations. This condition is met when the coefficient which multiplies $x$ in Eq. (10) is set to zero: $2 \alpha+\left(4-k^{2} / 2\right) \delta=0$. From this expression, for $\alpha=-0.2712$ and $k=1.772$, one can calculate $\delta=-4 \alpha /\left(8-k^{2}\right)=0.2232$. The solution so found is straight Talbot carpet, as shown in Fig. 6c. 
(a)

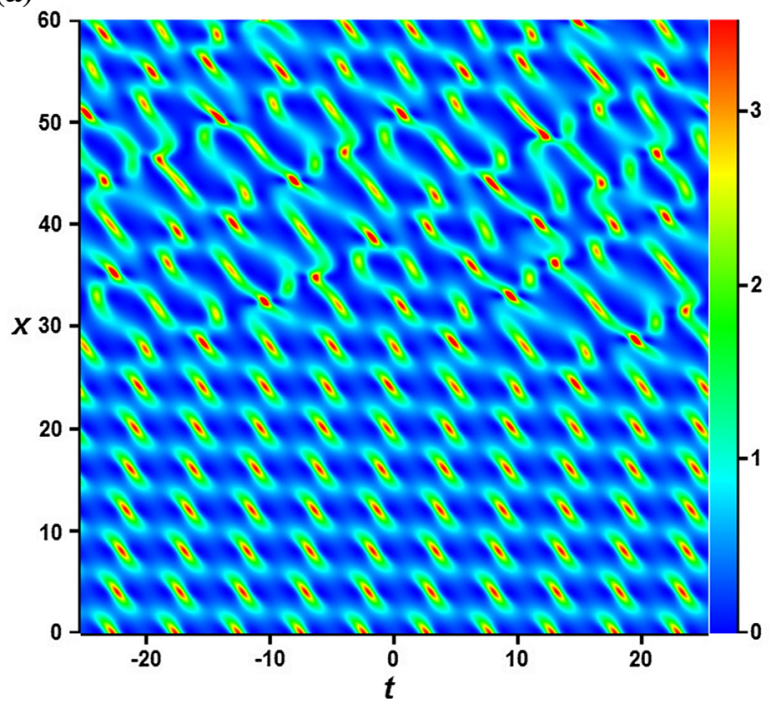

Fig. 5 Hirota Talbot carpet (numerical evolution) with 10 periods in a box. Initial conditions are given by analytical expression (9), with $\alpha=0.2712, \gamma=\delta=0$ and $k=1.7286$. a Normal evo- (b)

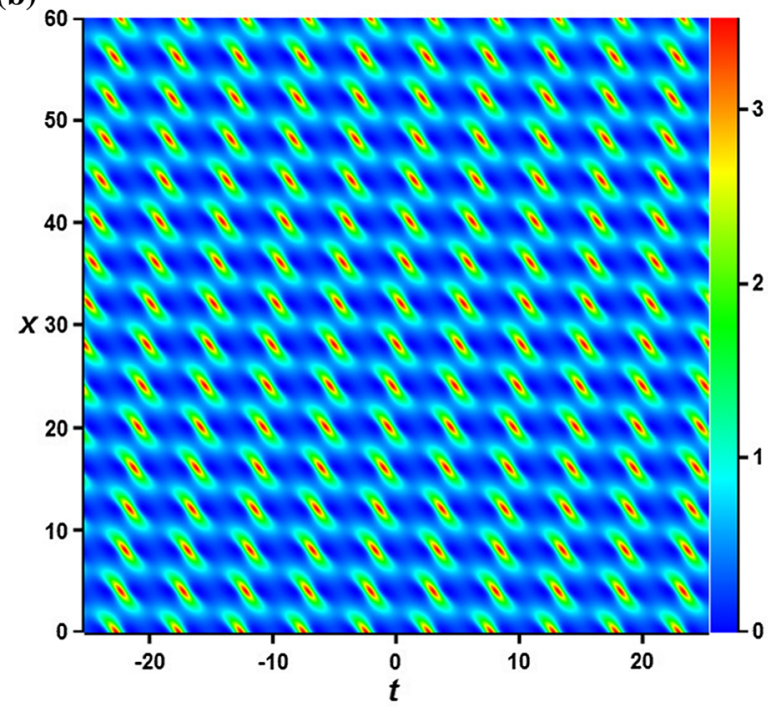

lution of the initial breather, with MI included. b Talbot carpet, with the pruning procedure applied (a)

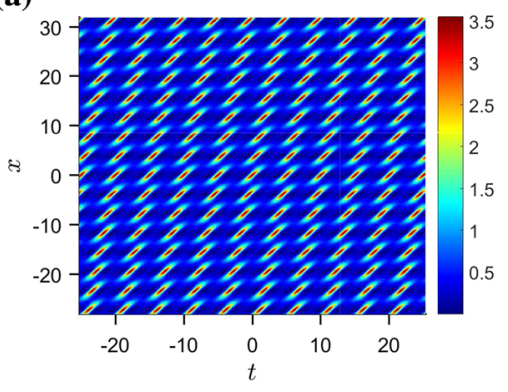

(b)

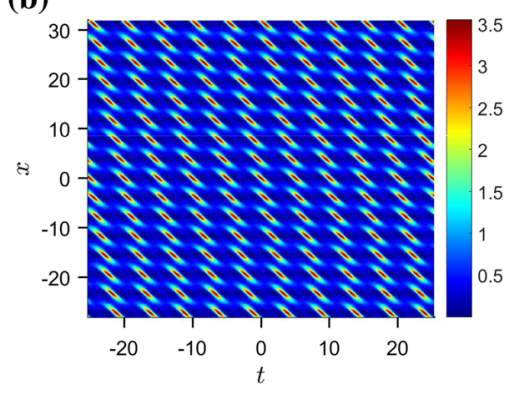

(c)

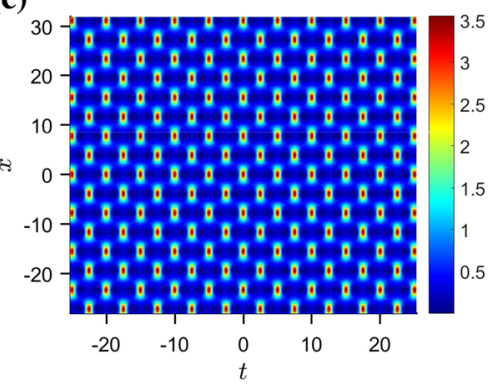

Fig. 6 Talbot carpet of Hirota-quintic equation $(\gamma=0$ and $k=1.772)$. Parameters are: $\mathbf{a} \alpha=-0.2712$ and $\delta=-0.1, \mathbf{b} \alpha=0.2712$ and $\delta=0.1, \mathbf{c} \alpha=-0.2712$ and $\delta=-\frac{4 \alpha}{8-k^{2}}=0.2232$

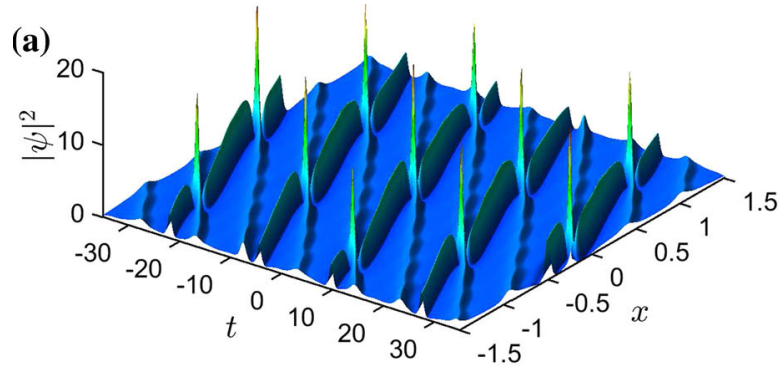

Fig.7 Quintic Talbot carpet made of the second-order breathers that are matched mutually and to the dn background wave. Parameters: $c=1, \alpha=-0.03, \gamma=-0.0614, \delta=0.7: \mathbf{a} m=0.1192$,

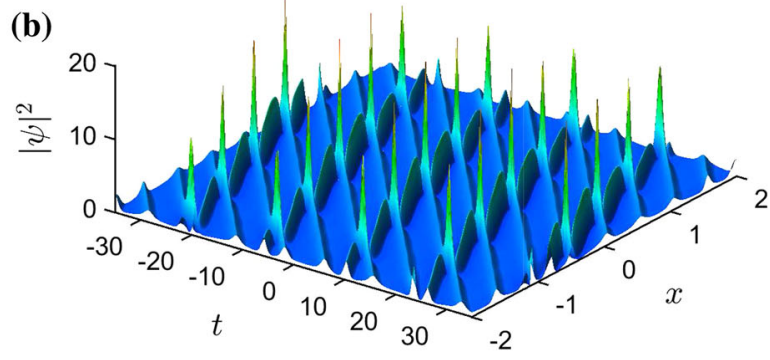

$\nu_{1}=0.92, \nu_{2}=0.83, q=9 . \mathbf{b} m=0.0505, \nu_{1}=0.92$, $\nu_{2}=0.8104, q=8$ 


\section{Double-periodic rogue waves of the quintic equation using period-matching procedure}

In this section, we discuss the numerical procedure for generating double-periodic RW solutions of the quintic equation $(\alpha \neq 0, \gamma \neq 0, \delta \neq 0)$. These are basically higher-order breathers obtained by the Darboux transformation technique, when one sets JEFs as the starting seed solutions $\psi_{0}(x, t)$. Being doubly periodic, they can appear in the form of Talbot carpets. Hence, no pruning procedure is necessary, owing to the extensive and very precise period matching that ensures constructive nonlinear interference of modes and prevents the growth of unstable sidebands. Only the fundamental double-periodic RW mode can grow. This is similar to the generation of the stable AB in Fig. 1, where also no pruning was necessary.

The discussion here leans a lot on the results obtained in [53]. We choose the dn function as the background $[7,8,53]$ :

$\psi_{0}(x, t)=c e^{i \phi x} \mathrm{dn}(c t+v x, m)$,

with $\phi=\frac{1}{2}(2-m) c^{2}+\gamma c^{4}\left(6-6 m+m^{2}\right)$ and $v=$ $(2-m) \alpha c^{3}+\delta c^{5}\left(6-6 m+m^{2}\right)$. Next, we calculate an arbitrary-order breather on the elliptic background, using the procedure described in detail in [53].

As shown in Sect. 5 of [53], the matching of the higher-order breather period $T_{B}$ to the period of the $\mathrm{dn}$ background $T_{d n}$ may lead to the appearance of periodic RWs. This means that the ratio of periods $q=T_{B} / T_{d n}$ should be a positive integer. In addition, it is also needed to match the periods $T_{B 1}, \ldots, T_{B K}$ of the DT constituents that form a $K$ th-order breather $\left(T_{B j}=T_{B 1} / j\right.$, for $j=2, \ldots, K$ ) with the background. An intricate period-matching procedure ensues, which leads to a Talbot carpet of periodic RWs that requires no pruning.

Two examples of the quintic Talbot carpets, consisting of the second-order breathers, are shown in Fig. 7. We take $c=1, \alpha=-0.03, \gamma=-0.0614$, and $\delta=0.7$. We use expressions from [53] to calculate $m=0.1192, v_{1}=0.92$, and $v_{2}=0.83$, leading to $q=9$ and $T_{B 2}=T_{B 1} / 2$. Here, $v_{1}$ and $\nu_{2}$ are the eigenvalues of the first two unstable modes, according to the DT. We get the double-periodic rogue wave shown in Fig. 7a. Similarly, we take the second set of values: $m=0.0505, v_{1}=0.92$, and $v_{2}=0.8104$, and obtain $q=8$. The result is another quintic Talbot carpet, seen in Fig. $7 b$.

\section{Conclusion}

In this paper, we have presented dynamical procedure for generating Talbot carpets from the solutions of generalized NLSEs, consisting of the first- and secondorder breathers. We have shown that to this end, it is necessary to calculate the initial wave for numerics using DT, and to set the transverse box size equal to an integer number of breather periods.

We have introduced two pruning algorithms, by which unstable Fourier modes that produce modulation instability and may ruin the carpet are eliminated or suppressed (except the ones building the fundamental breather).

We have next displayed new exact Talbot carpet solutions of the Hirota-quintic equation, which is a generalization of the NLSE resulting from a consistent Taylor expansion of dispersive and nonlinear terms. We have analyzed how the three parameters in the equation affect intensity distributions in the carpet. We pointed out that for particular combination of the parameter values, common solutions of both NLSE and Hirotaquintic equation can be obtained.

In the end, we applied the analysis from our previous work to generate doubly periodic rogue waves of the quintic equation. We used an extensive period matching of higher-order breathers and an elliptic background on which the breather is constructed, to produce rare periodic RWs which obliviate the need for using the pruning procedure to suppress MI.

We believe that various Talbot carpet solutions of extended nonlinear Schrödinger equations (up to the fifth-order dispersion) can find applications within the broad class of self-imaging phenomena and possibly for nano-photonic lithography. An interesting extension of this work is to invent pruning techniques that are amenable to experimental generation of modes in fibers, in which only the adverse effects of MI are suppressed.

Acknowledgements Open Access funding provided by the Qatar National Library. This research is supported by the Qatar National Research Fund (Project NPRP 8-028-1-001). S.N.N. acknowledges support from Grants III45016 and OI171038 of the Serbian Ministry of Education, Science and Technological Development. N.B.A. acknowledges support from Grant 
OI171006 of the Serbian Ministry of Education, Science and Technological Development and project No. 18-11-0024 of the Russian Science Foundation. M.R.B. acknowledges support by the Al-Sraiya Holding Group.

Open Access This article is distributed under the terms of the Creative Commons Attribution 4.0 International License (http://creativecommons.org/licenses/by/4.0/), which permits unrestricted use, distribution, and reproduction in any medium, provided you give appropriate credit to the original author(s) and the source, provide a link to the Creative Commons license, and indicate if changes were made.

\section{References}

1. Kivshar, Y.S., Agrawal, G.P.: Optical Solitons. Academic Press, San Diego (2003)

2. Agrawal, G.P.: Applications of Nonlinear Fiber Optics. Academic Press, San Diego (2001)

3. Fibich, G.: The Nonlinear Schrödinger Equation. Springer, Berlin (2015)

4. Dudley, J.M., Dias, F., Erkintalo, M., Genty, G.: Instabilities, breathers and rogue waves in optics. Nat. Photon. 8, 755 (2014)

5. Dudley, J.M., Taylor, J.M.: Supercontinuum Generation in Optical Fibers. Cambridge University Press, Cambridge (2010)

6. Solli, D.R., Ropers, C., Koonath, P., Jalali, B.: Optical rogue waves. Nature 450, 1054 (2007)

7. Ankiewicz, A., Kedziora, D.J., Chowdury, A., Bandelow, U., Akhmediev, N.: Infinite hierarchy of nonlinear Schrödinger equations and their solutions. Phys. Rev. E 93, 012206 (2016)

8. Kedziora, D.J., Ankiewicz, A., Chowdury, A., Akhmediev, N.: Integrable equations of the infinite nonlinear Schrödinger equation hierarchy with time variable coefficients. Chaos 25, 103114 (2015)

9. Chin, S.A., Ashour, O.A., Belić, M.R.: Anatomy of the Akhmediev breather: cascading instability, first formation time, and Fermi-Pasta-Ulam recurrence. Phys. Rev. E 92, $063202(2015)$

10. Bogolubov, N.: On the theory of superfluidity. J. Phys. (USSR) 11, 23 (1947)

11. Akhmediev, N.N., Korneev, V.I.: Modulation instability and periodic solutions of the nonlinear Schrödinger equation. Theor. Math. Phys. 69, 1089 (1986)

12. Akhmediev, N., Eleonskii, V., Kulagin, N.: Exact first-order solutions of the nonlinear Schrödinger equation. Theor. Math. Phys. 72, 809 (1987)

13. Erkintalo, M., Hammani, K., Kibler, B., Finot, C., Akhmediev, N., Dudley, J.M., Genty, G.: Higher-order modulation instability in nonlinear fiber optics. Phys. Rev. Lett. 107, 253901 (2011)

14. Chin, S.A., Ashour, O.A., Nikolić, S.N., Belić, M.R.: Maximal intensity higher-order Akhmediev breathers of the nonlinear Schrödinger equation and their systematic generation. Phys. Lett. A 380, 3625 (2016)
15. Herbst, B.M., Ablowitz, M.J.: Numerically induced chaos in the nonlinear Schrödinger equation. Phys. Rev. Lett. 62, 2065 (1989)

16. Ablowitz, M.J., Herbst, B.M.: On homoclinic structure and numerically induced chaos for the nonlinear Schrödinger equation. SIAM J. Appl. Math. 50, 339 (1990)

17. Calini, A., Schober, C.M.: Homoclinic chaos increases likelihood of rogue wave formation. Phys. Lett. A 298, 335 (2002)

18. Calini, A., Schober, C.M.: Dynamical criteria for rogue waves in nonlinear Schrödinger models. Nonlinearity $\mathbf{2 5}$, R99 (2012)

19. Toenger, S., Godin, T., Billet, C., Dias, F., Erkintalo, M., Genty, G., Dudley, J.M.: Emergent rogue wave structures and statistics in spontaneous modulation instability. Sci. Rep. 5, 10380 (2015)

20. Akhmediev, N., et al.: Roadmap on optical rogue waves and extreme events. J. Opt. 18, 063001 (2016)

21. Zhang, Y.Q., Belić, M.R., Zheng, H., Chen, H., Li, C., Song, J., Zhang, Y.P.: Nonlinear Talbot effect of rogue waves. Phys. Rev. E 89, 032902 (2014)

22. Zhang, Y., Belić, M.R., Petrović, M.S., Zheng, H., Chen, H., Li, C., Lu, K., Zhang, Y.: Two-dimensional linear and nonlinear Talbot effect from rogue waves. Phys. Rev. E 91, 032916 (2015)

23. Talbot, H.F.: Facts relating to optical science. Philos. Mag. 9, 401 (1836)

24. Wen, J., Du, S., Chen, H., Xiao, M.: Electromagnetically induced Talbot effect. Appl. Phys. Lett. 98, 081108 (2011)

25. Zhang, Y.Q., Yao, X., Yuan, C.Z., Li, P.Y., Yuan, J.M., Feng, W.K., Jia, S.Q., Zhang, Y.P.: Controllable multiwave mixing Talbot effect. IEEE Photon. J. 4, 2057 (2012)

26. Song, X.-B., Wang, H.-B., Xiong, J., Wang, K., Zhang, X., Luo, K.-H., Wu, L.-A.: Experimental observation of quantum Talbot effects. Phys. Rev. Lett. 107, 033902 (2011)

27. Iwanow, R., May-Arrioja, D.A., Christodoulides, D.N., Stegeman, G.I., Min, Y., Sohler, W.: Discrete Talbot effect in waveguide arrays. Phys. Rev. Lett. 95, 053902 (2005)

28. Deng, L., Hagley, E.W., Denschlag, J., Simsarian, J.E., Edwards, M., Clack, C.W., Helmerson, K., Rolston, S.L., Phillips, W.D.: Temporal, matter-wave-dispersion Talbot effect. Phys. Rev. Lett. 83, 5407 (1999)

29. Ryu, C., Andersen, M.F., Vaziri, A., d'Arcy, M.B., Grossman, J.M., Helmerson, K., Phillips, W.D.: High-order quantum resonances observed in a periodically kicked BoseEinstein condensate. Phys. Rev. Lett. 96, 160403 (2006)

30. Ramezani, H., Christodoulides, D.N., Kovanis, V., Vitebskiy, I., Kottos, T.: PT-symmetric Talbot effects. Phys. Rev. Lett. 109, 033902 (2012)

31. Pfeiffer, F., Bech, M., Bunk, O., Kraft, P., Eikenberry, E.F., Brönnimann, C., Grünzweig, C., David, C.: Hard-Xray dark-field imaging using a grating interferometer. Nat. Mater. 7, 134 (2008)

32. Zhang, Y., Wen, J., Zhu, S.N., Xiao, M.: Nonlinear Talbot effect. Phys. Rev. Lett. 104, 183901 (2010)

33. Wen, J., Zhang, Y., Xiao, M.: The Talbot effect: recent advances in classical optics, nonlinear optics, and quantum optics. Adv. Opt. Photon. 5, 83 (2013)

34. Ashour O.A.: Maximal Intensity Higher-Order Breathers of the Nonlinear Schrödinger Equation on Different Back- 
grounds. Undergraduate Research Scholars Thesis, Texas A\&M University, USA (2017)

35. Chowdury, A., Kedziora, D.J., Ankiewicz, A., Akhmediev, N.: Breather-to-soliton conversions described by the quintic equation of the nonlinear Schrödinger hierarchy. Phys. Rev. E 91, 032928 (2015)

36. Trippenbach, M., Band, Y.B.: Effects of self-steepening and self-frequency shifting on short-pulse splitting in dispersive nonlinear media. Phys. Rev. A 57, 4791 (1998)

37. Wang, D.-S., Chen, F., Wen, X.-Y.: Darboux transformation of the general Hirota equation: multisoliton solutions, breather solutions and rogue wave solutions. Adv. Differ. Equ. 2016, 67 (2016)

38. Guo, R., Hao, H.Q.: Breathers and multi-solitons solutions for the higher-order generalized nonlinear Schrödinger equation. Commun. Nonlinear Sci. Numer. Simul. 18, 24262435 (2013)

39. Mani Rajan, M.S., Mahalingam, A.: Nonautonomous solitons in modified inhomogeneous Hirota equation: soliton control and soliton interaction. Nonlinear Dyn. 79, 24692484 (2015)

40. Anderson, D., Lisak, M.: Nonlinear asymmetric self-phase modulation and self-steepening of pulses in long optical waveguides. Phys. Rev. A 27, 1393 (1983)

41. Backus, S., Durfee III, C.G., Mourou, G., Kapteyn, H.C., Murnane, M.M.: 0.2-TW laser system at $1 \mathrm{kHz}$. Opt. Lett. 22, 1256 (1997)

42. Mirzazadeh, M., Eslami, M., Zerrad, E., Mahmood, M.F., Biswas, A., Belić, M.: Optical solitons in nonlinear directional couplers by sine-cosine function method and Bernoulli's equation approach. Nonlinear Dyn. 81, 19331349 (2015)

43. Biswas, A., Khalique, C.M.: Stationary solutions for nonlinear dispersive Schroödinger equation. Nonlinear Dyn. 63, 623-626 (2011)

44. Hirota, R.: Exact envelope-soliton solutions of a nonlinear wave equation. J. Math. Phys. 14, 805-809 (1973)

45. Tao, Y., He, J.: Multisolitons, breathers, and rogue waves for the Hirota equation generated by the Darboux transformation. Phys. Rev. E 85, 026601 (2012)
46. Guo, R., Zhao, X.-J.: Discrete Hirota equation: discrete Darboux transformation and new discrete soliton solutions. Nonlinear Dyn. 84, 1901-1907 (2016)

47. Nikolić, S.N., Aleksić, N.B., Ashour, O.A., Belić, M.R., Chin, S.A.: Systematic generation of higher-order solitons and breathers of the Hirota equation on different backgrounds. Nonlinear Dyn. 89, 1637-1649 (2017)

48. Chowdury, A., Kedziora, D.J., Ankiewicz, A., Akhmediev, N.: Soliton solutions of an integrable nonlinear Schrödinger equation with quintic terms. Phys. Rev. E 90, 032922 (2014)

49. Lan, Z., Gao, B.: Solitons, breather and bound waves for a generalized higher-order nonlinear Schrödinger equation in an optical fiber or a planar waveguide. Eur. Phys. J. Plus 132, 512 (2017)

50. Chowdury, A., Kedziora, D.J., Ankiewicz, A., Akhmediev, N.: Breather solutions of the integrable nonlinear Schrödinger equation and their interactions. Phys. Rev. E 91, 022919 (2015)

51. Yang, Y., Yan, Z., Malomed, B.A.: Rogue waves, rational solitons, and modulational instability in an integrable fifthorder nonlinear Schrödinger equation. Chaos 25, 103112 (2015)

52. Kedziora, D.J., Ankiewicz, A., Akhmediev, N.: Circular rogue wave clusters. Phys. Rev. E 84, 056611 (2011)

53. Nikolić, S.N., Ashour, O.A., Aleksić, N.B., Belić, M.R., Chin, S.A: Breathers, solitons and rogue waves of the quintic nonlinear Schrödinger equation on various backgrounds. Nonlinear Dyn. 95, 2855-2865 (2019)

Publisher's Note Springer Nature remains neutral with regard to jurisdictional claims in published maps and institutional affiliations. 